\title{
First and second order Opial inequalities
}

by

STEVEN BLOOM (Loudonville, N.Y.)

Abstract. Let $T_{\gamma} f(x)=\int_{0}^{x} k(x, y)^{\gamma} f(y) d y$, where $k$ is a nonnegative kernel increasing n $x$, decreasing in $y$, and satisfying a triangle inequality. An $n$ th-order Opial inequality has the form

$$
\int_{0}^{\infty}\left(\prod_{i=1}^{n}\left|T_{\gamma_{i}} f(x)\right|^{q_{i}}\right)|f(x)|^{q_{0}} w(x) d x \leq C\left(\int_{0}^{\infty}|f(x)|^{p} v(x) d x\right)^{\left(q_{0}+\cdots+q_{n}\right) / p} .
$$

Such inequalities can always be simplified to $n$th order reduced inequalities, where the exponent $q_{0}=0$. When $n=1$, the reduced inequality is a standard weighted norm inequality, and characterizing the weights is easy. We also find necessary and sufficient conditions on the weights for second-order reduced Opial inequalities to hold.

1. Introduction. Opial's inequality is the elementary inequality

$$
\int_{0}^{a}\left|f(x) f^{\prime}(x)\right| d x \leq \frac{a}{2} \int_{0}^{a}\left|f^{\prime}(x)\right|^{2} d x
$$

when $f(0)=0$. This was proven by Opial [13] in 1960 . Since then, numerous authors have studied variants of this. The most natural extension is the weighted Opial inequality

$$
\int_{a}^{b}|f(x)|^{q}\left|f^{\prime}(x)\right|^{r} w(x) d x \leq C\left(\int_{a}^{b}\left|f^{\prime}(x)\right|^{p} v(x) d x\right)^{(q+r) / p}
$$

when $f(a)=0$. There are two fundamental questions associated with (2).

- The Existence Question: For what weights $(v, w)$ and exponents $(p ; q, r)$ will (2) hold?

and

- The Optimization Question: If (2) does hold for a given pair of weights and a range of exponents, what is the best constant $C$ ?

1991 Mathematics Subject Classification: 26A33, 26D15.

Research supported in part by a grant from Siena College. 
Best constant problems were more or less completely settled in Boyd's beautiful papers [5], [6], but existence is still open.

Another generalization of (1) is to higher order derivatives, what we call an $n$ th-order Opial inequality:

(3) $\quad \int_{a}^{b} \prod_{i=0}^{n}\left|f^{(i)}(x)\right|^{q_{i}} w(x) d x \leq C\left(\int_{a}^{b}\left|f^{(n)}(x)\right|^{p} v(x) d x\right)^{\left(q_{0}+\ldots+q_{n}\right) / p}$

where here, $f(a)=f^{\prime}(a)=\ldots=f^{(n-1)}(a)=0$.

Several authors have studied existence questions for (3), notably [8], [9], and [14], and each gave sufficient conditions on the weights for (3) to hold. Likewise, there have been many papers detailing sufficient conditions for (2), such as [2], [3], and [18]. The sharpest conditions for the first order inequality are due to Sinnamon, who proceeded from the following simple observation: Let

$$
I f(x)=\int_{0}^{x} f(t) d t
$$

This is the Hardy anti-differentiation operator. Then (2) can be rewritten as

$$
\int_{0}^{b}|f(x)|^{r}|I f(x)|^{q} w(x) d x \leq C\left(\int_{0}^{b}|f(x)|^{p} v(x) d x\right)^{(q+r) / p} .
$$

Applying Hölder's inequality, when $r<p$, we get

$$
\int_{0}^{b}|f|^{r}|I f|^{q} w d x \leq\left(\int_{0}^{b}|f|^{p} v d x\right)^{r / p}\left(\int_{0}^{b}|I f|^{q(p / r)^{\prime}} w^{(p / r)^{\prime}} v^{-r /(p-r)} d x\right)^{1-r / p}
$$

where the prime represents the conjugate exponent, i.e., $1 / s+1 / s^{\prime}=1$. So a sufficient condition for (2) to hold can be obtained by choosing weights for which

$$
\int_{0}^{b}|I f|^{q(p / r)^{\prime}} w^{(p / r)^{\prime}} v^{-r /(p-r)} d x \leq C\left(\int_{0}^{b}|f|^{p} v d x\right)^{q(p / r)^{\prime} / p}
$$

(4) is, of course, just a special case of (2), with $r=0$ (and with different weights, and different exponents).

The inequality (3) with $q_{n}=0$ is known as a reduced $n$ th-order Opial inequality. Hölder's inequality shows that sufficient conditions for (3) can always be obtained from a corresponding reduced inequality, when $q_{n}<p$ (and even when $q_{n}=p$, with the reduced inequality on $L^{\infty}$ ).

This reduction when $n=1$ is to a weighted norm inequality for the Hardy operator $I$. In general, given an operator $T$ and a pair of weights $(v, w)$, a weighted norm inequality has the form

$$
\int|T f(x)|^{q} w(x) d x \leq C\left(\int|f(x)|^{p} v(x) d x\right)^{q / p}
$$

The weight problem is to characterize all weight pairs $(v, w)$ for which (5) holds. When $1<p, q<\infty$, the weight problem for the Hardy operator is solved ([1], [7], [11], [12], [15], [21], and [22]). For instance, when $1<p \leq q$,

$$
\int|I f(x)|^{q} w(x) d x \leq C\left(\int|f(x)|^{p} v(x) d x\right)^{q / p}
$$

holds if and only if

$$
I \sigma(x)^{q / p^{\prime}} I^{*} w(x) \leq C
$$

for all $x>0$. Here $I^{*}$ is the adjoint operator, $I^{*} g(x)=\int_{x}^{\infty} g(t) d t$, and $\sigma=v^{-p^{\prime} / p}$.

This $\sigma$ that appears in (6) arises in a very fundamental way. If we are studying an operator $T$ with kernel $k(x, y)$, it is quite natural to ask on what measure spaces is this operator $L^{p}-L^{q}$ bounded. For instance, if $\mu, \nu$ are measures on $\mathbb{R}^{n}$, we set

$$
T_{\nu} f(x)=\int_{\mathbb{R}^{n}} k(x, y) f(y) d v(y)
$$

and we are asking whether $T_{\nu}: L^{p}\left(\mathbb{R}^{n}, d \nu\right) \rightarrow L^{q}\left(\mathbb{R}^{n}, d \mu\right)$,

$$
\int_{\mathbb{R}^{n}}\left|T_{\nu} f(x)\right|^{q} d \mu(x) \leq C\left(\int_{\mathbb{R}^{n}}|f(x)|^{p} d \nu(x)\right)^{q / p}
$$

When these are absolutely continuous measures, $d \mu=w(x) d x, d \nu=$ $\sigma(x) d x$, then (7) is exactly equivalent to (5) with $\sigma=v^{-p^{\prime} / p}\left({ }^{1}\right)$. It is almost axiomatic, therefore, that any weight problem solution like (6) will be given in terms of $w$ and $\sigma$, not in terms of $v$.

When we refer to a weight pair $(v, w)$, we mean that the functions $w, \sigma$ are nonnegative and finite almost everywhere.

The weight conditions obtained by Sinnamon turn out to be necessary as well as sufficient. This is because the reduction process used by Sinnamon is actually sharp.

We call an operator $T$ positive if, whenever $0 \leq f \leq g$, we have

$$
0 \leq T f(x) \leq T g(x)
$$

for all $x$. Our first result is

(1) This observation is due to Sawyer [16], and has been a key starting point for his solution to several weight problems ([16], [17], and [10]). 
THEOREM 1. Let $T$ be a positive operator acting on a nonatomic $\sigma$-finite measure space $(X, d \mu)$, with $T \not \equiv 0$. Let $r, q, p>0$. Then

$$
\int_{X}|f(x)|^{r}|T f(x)|^{q} d \mu(x) \leq C\left(\int_{X}|f(x)|^{p} d \mu\right)^{(r+q) / p}
$$

can hold only if $r \leq p$, and in that case, (8) is equivalent to

$$
T: L^{p}(X, d \mu) \rightarrow L^{q(p / r)^{\prime}}(X, d \mu)
$$

We will extend the Opial inequalities (2) and (3) to the generalized Hardy operators introduced in [4].

Definitron 2. An operator $T$ acting on $\mathbb{R}^{+}$is called a generalized Hardy operator, or GHO, if $T$ has the form

$$
T f(x)=\int_{0}^{x} k(x, y) f(y) d y
$$

where the kernel $k \geq 0$ is nondecreasing in $x$, nonincreasing in $y$, and satisfies a triangle inequality

$$
k(x, y) \leq C[k(x, z)+k(z, y)]
$$

whenever $y<z<x$.

These include the Hardy operator and all higher order anti-derivatives, as well as the Riemann-Liouville fractional integrals,

$$
I_{\alpha} f(x)=\int_{0}^{x}(x-y)^{\alpha} f(y) d y
$$

We will write $T_{\gamma}$ for the operator

$$
T_{\gamma} f(x)=\int_{0}^{x} k(x, y)^{\gamma} f(y) d y
$$

and we write $T^{*}$ and $T_{\gamma}^{*}$ for the adjoint operators

$$
T_{\gamma}^{*} f(x)=\int_{x}^{\infty} k(y, x)^{\gamma} f(y) d y
$$

Notice that $T_{0}$ is the Hardy operator $I$.

Let $1<p<\infty, \gamma_{i}, q_{i} \geq 0$, and let $(v, w)$ be a weight pair. The $n$ th-order Opial inequality is

(10) $\quad \int_{0}^{\infty}\left(\prod_{i=1}^{n}\left|T_{\gamma_{i}} f(x)\right|^{q_{i}}\right)|f(x)|^{q_{0}} w(x) d x \leq C\left(\int_{0}^{\infty}|f|^{p} v d x\right)^{\left(q_{0}+\ldots+q_{n}\right) / p}$ and an $n$ th-order reduced Opial inequality is (10) with $q_{0}=0 .(10)$ can be rewritten, à la Sawyer, as

$\int_{0}^{\infty}\left(w \cdot \sigma^{q_{0}-1} \prod_{i=1}^{n}\left|T_{\gamma_{i}} \sigma \cdot f\right|^{q_{i}}\right)|f(x)|^{q_{0}} \sigma(x) d x \leq C\left(\int_{0}^{\infty}|f|^{p} \sigma d x\right)^{\left(q_{0}+\ldots+q_{n}\right) / p}$

and since $\left(w \cdot \sigma^{q_{0}-1} \prod_{i=1}^{n}\left|T_{\gamma_{i}} \sigma \cdot\right|^{q_{i}}\right)^{1 /\left(q_{1}+\ldots+q_{n}\right)}$ is a positive (although nonlinear) operator, we can apply Theorem 1 . And so, (10) is equivalent to the corresponding $n$ th-order reduced Opial inequality.

The first order Opial inequality reduces to a weighted norm inequality (5) for a GHO $T$. For such operators and $p>1, q \geq 1$, the weight problem is completely solved ([4], [19], [20], [10]), and so we have a complete solution to the existence question for $(10)$ when $q_{1}\left(p / q_{0}\right)^{\prime} \geq 1$. We will prove Theorem $1 \mathrm{in}$ the next section and present this existence solution for first order Opial inequalities.

Sufficient conditions for (3) to hold with $n \geq 2$ have been obtained in [8], [9], and [14]. The best of these is Li's paper [9], where the following is shown: If $p=\sum q_{i}, q_{n}>0$, set

$$
\begin{gathered}
Q=\prod_{i=0}^{n-1}[(n-1-i) !]^{q_{i}} \\
R_{i}(x)=\left[\int_{a}^{x}(x-t)^{(n-1-i) p^{\prime}} \sigma(t) d t\right]^{q_{i}(p-1) /\left(p-q_{n}\right)}
\end{gathered}
$$

and

$$
K_{n}(v, w)=\left[\int_{a}^{b} w(x)^{p /\left(p-q_{n}\right)} v(x)^{-q_{n} /\left(p-q_{n}\right)} \prod_{i=0}^{n-1} R_{i}(x) d x\right]^{\left(p-q_{n}\right) / p}
$$

Then (3) holds with

$$
C=\frac{K_{n}(v, w)}{Q}\left(\frac{q_{n}}{p}\right)^{q_{n} / p}\left({ }^{2}\right)
$$

Unfortunately, this condition is quite far from necessary. Indeed, perhaps the simplest weighted Opial inequality is

$$
\int_{0}^{1}\left|f \cdot f^{\prime}\right| \frac{d x}{x} \leq C \int_{0}^{1}\left|f^{\prime}\right|^{2} d x
$$

which follows trivially from the classical Hardy inequality. But here, Li's constant $K_{1}(1,1 / x)$ is infinite. The best known results are not sharp enough to reproduce even very simple inequalities like (11).

$\left({ }^{2}\right)$ This result is stronger than the results proven by Cheung or Pachpatte. Theorem 1 in [14], for instance, is simply a special case of Li's theorem. 
Thanks to Theorem 1, the study of an $n$ th-order Opial inequality can be simplified to the corresponding $n$ th-order reduced Opial inequality. The remainder of the paper is devoted to the study of the second order reduced inequality

(12) $\int\left|T_{\gamma_{0}} f(x)\right|^{q_{0}}\left|T_{\gamma_{2}} f(x)\right|^{q_{1}} w(x) d x \leq C\left(\int|f(x)|^{p} v(x) d x\right)^{\left(q_{0}+q_{1}\right) / p}$

when $1<p \leq q_{0}+q_{1}, q_{0}, q_{1} \geq 1$.

The restriction $p \leq q_{0}+q_{1}$ corresponds to the usual Riesz triangle in operator theory, but here, surprisingly, the situation is much easier if $p \leq$ $q_{0}, q_{1}$.

In Section 3, we will characterize the corresponding weak-type inequality. Obviously,

$\int\left|T_{\gamma_{0}} f(x)\right|^{q_{0}}\left|T_{\gamma_{1}} f(x)\right|^{q_{1}} w(x) d x \geq \lambda_{0}^{q_{0}} \lambda_{1}^{q_{1}} w\left(\left[x:\left|T_{\gamma_{i}} f(x)\right|>\lambda_{i}\right.\right.$ for $\left.\left.i=0,1\right]\right)$.

We prove the following:

THEOREM 3. Let $S$ and $T$ be linear operators with kernels $j(x, y)$ and $k(x, y)$ respectively on $\mathbb{R}^{+} \times \mathbb{R}^{+}$, which are nonnegative and nondecreasing in $x$. Then the weak-type Opial inequality

$$
\lambda^{r} \mu^{q} w([x:|T f(x)|>\lambda,|S f(x)|>\mu]) \leq C\left(\int|f|^{p} v\right)^{(q+r) / p}
$$

holds for $q, r>0, p>1$, and $(v, w)$ a weight pair, if and only if

$$
S_{p^{\prime}} \sigma(x)^{q / p^{\prime}} T_{p^{\prime}} \sigma(x)^{r / p^{\prime}} I^{*} w(x) \leq C
$$

for all $x>0$.

In Section 4, we begin our study of the second order Opial inequalities in earnest. The previous results are all quite straightforward, but things begin to get very technical here. The general battle plan is this: We can simplify a second order Opial inequality to a second order reduced inequality (12). That can be reduced even further to a special case where one of the kernel exponents $\gamma_{i}$ is zero. Then, by a careful use of the triangle inequality for the remaining kernel, this can be reduced to a series of first order problems, and those we can solve. So the scheme is

$$
\begin{aligned}
\text { 2nd-order } & \rightarrow \text { 2nd-order reduced } \\
& \rightarrow \text { special case: } \gamma_{0}=0 \\
& \rightarrow \text { st-order } \\
& \rightarrow \text { known weighted norm inequalities. }
\end{aligned}
$$

This scheme governs the remainder of the paper. We begin with a large collection of necessary conditions. These are all presented in Section 4. In
Section 5 , we deal with the special case. We investigate the inequality

$$
\int_{0}^{\infty}|I f(x)|^{q}|T f(x)|^{r} w(x) d x \leq C\left(\int_{0}^{\infty}|f(x)|^{p} v(x) d x\right)^{(q+r) / p} .
$$

The case when $p \leq q$ is much simpler, and there we prove:

THEOREM 4. Let $1<p \leq q<\infty, 1 \leq r<\infty$, let $(v, w)$ be a weight pair, and let $T$ be a GHO. Then (15) holds if and only if both of the following conditions hold: For each $x>0$,

and

$$
I \sigma(x)^{q / p^{\prime}} T_{p^{\prime}} \sigma(x)^{r / p^{\prime}} I^{*} w(x) \leq C
$$

For $q<p$, we obtain:

THEOREM 5. Let $1<p<\infty, 1 \leq q, r<\infty$ with $q<p \leq q+r$. Let $T$ be $a$ GHO and let $(v, w)$ be a weight pair. Then the following are equivalent:

(i) The Opial inequality (15) holds.

(ii) Four separate conditions hold. For each $x>0$, we have (16), (17), as well as

$$
\text { (18) } \quad T_{p^{\prime}} \sigma(x)^{\left(r / p^{\prime}\right)(p / q)^{\prime}} \int_{x}^{\infty} \sigma(y)\left[I^{*} w(y) I \sigma(y)^{q-1}\right]^{(p / q)^{\prime}} d y \leq C
$$

and

$$
\text { (19) } \quad I \sigma(x)^{\left(r / p^{\prime}\right)(p / q)^{\prime}} \int_{x}^{\infty} k(y, x)^{r(p / q)^{\prime}} \sigma(y)\left[I^{*} w(y) I \sigma(y)^{q-1}\right]^{(p / q)^{\prime}} d y \leq C .
$$

(iii) Four separate conditions hold. For each $x>0$, we have (16), (17), as well as

$$
T_{p^{\prime}} \sigma(x)^{\left(r / p^{\prime}\right)(p / q)^{\prime}} \int_{x}^{\infty} w(y)\left[I^{*} w(y) I \sigma(y)^{p-1}\right]^{(p / q)^{\prime}-1} d y \leq C
$$

and

(21) $I \sigma(x)^{\left(r / p^{\prime}\right)(p / q)^{\prime}} \int_{x}^{\infty} k(y, x)^{r(p / q)^{\prime}} w(y)\left[I^{*} w(y) I \sigma(y)^{p-1}\right]^{(p / q)^{\prime}-1} d y \leq C$.

Finally, in the last section, we tackle (12). By redefining the kernel, it suffices to consider $\gamma_{0}=1, \gamma_{1}>1$. We show

THEOREM 6 (Main Theorem). Let $r, q \geq 1,1<p \leq q+r$. Let $T$ be $a$ GHO, $\gamma>1$, and let $(v, w)$ be a weight pair. Then the Opial inequality

(22) $\quad \int_{0}^{\infty}\left|T^{\prime} f(x)\right|^{q}\left|T_{\gamma} f(x)\right|^{r} w(x) d x \leq C\left(\int_{0}^{\infty}|f(x)|^{p} v(x) d x\right)^{(q+r) / p}$ 
holds if and only if each of the following hold: For each $x>0$, we have

and

$$
\begin{aligned}
T_{p^{\prime}} \sigma(x)^{q / p^{\prime}} T_{\gamma p^{\prime}} \sigma(x)^{r / p^{\prime}} I^{*} w(x) & \leq C, \\
I \sigma(x)^{q / p^{\prime}} T \gamma_{p^{\prime}} \sigma(x)^{r / p^{\prime}} T_{q}^{*} w(x) & \leq C,
\end{aligned}
$$

If, in addition, $q<p$, then we also have the conditions

(26) $\quad T_{\gamma p^{\prime}} \sigma(x)^{\left(r / p^{\prime}\right)(p / q)^{\prime}} I^{*}\left[w\left\{I^{*} w\left(T_{p^{\prime}} \sigma\right)^{p-1}\right\}^{(p / q)^{\prime}-1}\right](x) \leq C$,

(27) $\quad I \sigma(x)^{\left(r / p^{\prime}\right)(p / q)^{\prime}} T_{\gamma r(p / q)^{\prime}}^{*}\left[w\left\{I^{*} w\left(T_{p^{\prime}} \sigma\right)^{p-1}\right\}^{(p / q)^{\prime}-1}\right](x) \leq C$,

(28) $\quad T_{\gamma p^{\prime}} \sigma(x)^{\left(r / p^{\prime}\right)(p / q)^{\prime}} I^{*}\left[\sigma\left\{T_{q}^{*} w(I \sigma)^{q-1}\right\}^{(p / q)^{\prime}}\right](x) \leq C$,

and

(29) $\quad I \sigma(x)^{\left(r / p^{\prime}\right)(p / q)^{\prime}} T_{\gamma r(p / q)}^{*}\left[\sigma\left\{T_{q}^{*} w(I \sigma)^{q-1}\right\}^{(p / q)^{\prime}}\right](x) \leq C$.

Finally, if $r<p$, then we also have the conditions

(30) $\quad T_{p^{\prime}} \sigma(x)^{\left(q / p^{\prime}\right)(p / r)^{\prime}} I^{*}\left[w\left\{I^{*} w\left(T_{\gamma p^{\prime}} \sigma\right)^{p-1}\right\}^{(p / r)^{\prime}-1}\right](x) \leq C$

and

(31) $\quad I \sigma(x)^{\left(q / p^{\prime}\right)(p / r)^{\prime}} T_{q(p / r)^{\prime}}^{*}\left[w\left\{I^{*} w\left(T_{\gamma p^{\prime}} \sigma\right)^{p-1}\right\}^{(p / r)^{\prime}-1}\right](x) \leq C$.

Throughout this paper, we follow the notational convention that $C$ will stand for a universal constant, which may vary from line to line, even within a string of inequalities.

2. First order Opial inequalities. The key here is the reduction given by Theorem 1 . We start with that.

Proof of Theorem 1. Suppose that (8) holds. Since $T$ is nontrivial, there exists an $f \in L^{p}$ with $f \geq 0$ and $T f \not \equiv 0$. So $T f \geq \varepsilon>0$ on a set $E$ of positive and finite measure. We normalize $f$ so that $\|f\|_{p}=1$. Let $0 \leq g$ be supported on $E$ with $\|g\|_{p} \leq 1$. Then (8) gives

$$
\int(f+g)^{r} T(f+g)^{q} d \mu(x) \leq 2^{r+q} C .
$$

Hence,

$$
\int g^{r} d \mu \leq \frac{2^{r+q} C}{\varepsilon^{q}}
$$

Thus $L^{p}(E) \subset L^{r}(E)$, which forces $r \leq p$.

Likewise (8) gives for $f \geq 0,\|f\|_{p} \leq 1$,

$$
\sup _{\|g\|_{p} \leq 1, g \geq 0} \int_{X} g^{r}(T f)^{q} d \mu \leq 2^{r+q} C
$$

which gives (9). The converse is Hölder's inequality.
To apply this theorem to first order Opial inequalities, we need the corresponding weighted norm inequalities for generalized Hardy operators. For that, we use Stepanov's theorem $\left({ }^{3}\right)[20]$ :

THEOREM 7. Let $1<p<\infty, T$ be a GHO, and $(v, w)$ a weight pair. Then $T: L^{p}(v) \rightarrow L^{q}(w)$ if and only if we have, either

(i) $p \leq q<\infty$ and both

and

$$
T_{p^{\prime}} \sigma(x)^{q / p^{\prime}} I^{*} w(x) \leq C
$$

for all $x>0$, or
(ii) $1 \leq q<p$ and both

$$
\int_{0}^{\infty} \sigma(t)\left[T_{q}^{*} w(t) I \sigma(t)^{q-1}\right]^{(p / q)^{\prime}} d t \leq C
$$

and

$$
\int_{0}^{\infty} w(t)\left[I^{*} w(t) T_{p^{\prime}} \sigma(t)^{p-1}\right]^{(p / q)^{\prime}-1} d t \leq C .
$$

Applying Theorem 1, the Opial inequality

(36) $\quad \int_{0}^{\infty}|f(x)|^{r}|T f(x)|^{q} w(x) d x \leq C\left(\int_{0}^{\infty}|f(x)|^{p} v(x) d x\right)^{(q+r) / p}$

holds when $p>1,0<r, q$, and $r<p$, if and only if

$$
T: L^{p}(v) \rightarrow L^{q(p / r)^{\prime}}\left[\left(w^{p} v^{-r}\right)^{1 /(p-r)}\right] .
$$

If $p \leq q+r$, then $q(p / r)^{\prime} \geq p$, and so we have:

Corollary 8. Let $T$ be a GHO, $(v, w)$ a weight pair, $q, r>0$, and $1<p \leq q+r$. Then the first order Opial inequality (36) holds if and only if both

$$
T_{p^{\prime}} \sigma(x)^{\left(q / p^{\prime}\right)(p / r)^{\prime}} \int_{x}^{\infty}\left[w^{p} v^{-r}\right]^{1 /(p-r)} d y \leq C
$$

and

$$
I \sigma(x)^{\left(q / p^{\prime}\right)(p / r)^{\prime}} \int_{x}^{\infty} k(y, x)^{q(p / r)^{\prime}}\left[w^{p} v^{-r}\right]^{1 /(p-r)} d y \leq C .
$$

$\left({ }^{3}\right)$ Calling this Stepanov's theorem is perhaps a bit unfair. Necessary and sufficient conditions also appeared in two other papers, [4] and [10], and both of these papers contributed greatly to the final form of this theorem. Still, the conditions (32)-(35) are Stepanov's, and he is the only author to deal with the full range of $q$. 
When $p>q+r, q(p / r)^{\prime}<p$. To apply Stepanov's theorem here, we need this $\geq 1$, or $p \leq q p+r$. That gives:

CoROllary 9. Let $T$ be a GHO, $(v, w)$ a weight pair, $q, r>0$, and $1<p$. If $q+r<p<q p+r$, then the first order Opial inequality (36) holds if and only if we have both

(40) $\int_{0}^{\infty} \sigma(t)\left[T_{q(p / r)^{\prime}}^{*}\left(w^{p /(p-r)} v^{-r /(p-r)}\right)(t) I \sigma(t)^{q(p / r)^{\prime}-1}\right]^{\left(p / q(p / r)^{\prime}\right)^{\prime}} d t \leq C$

and

(41) $\quad \int_{0}^{\infty} w(t)\left[I^{*}\left(w^{p /(p-r)} v^{-r /(p-r)}\right)(t) T_{p^{\prime}} \sigma(t)^{p-1}\right]^{\left(p / q(p / r)^{\prime}\right)^{\prime}-1} d t \leq C$.

When $p=q p+r$, then (36) holds if and only if (40).

When the kernel $k(x, y)=\chi_{(0, x)}(y)$, then $T$ is the Hardy operator $I$. In that case, (38) and (39) are equivalent, as are (40) and (41), and these conditions are the (sufficient) conditions given by Sinnamon. Sinnamon also extends the Hardy theorem to the case $q<1$, and so extends Corollary 9 .

3. Second order reduced inequalities: weak type. We now turn to the second order reduced inequality, and establish the weak type conditions of Theorem 3.

Proof of Theorem 3. Since the kernels are increasing, if we fix our attention on an $f>0$, the set where $T f(x)>\lambda$ will be an interval of the form $(a, \infty)$ or $[a, \infty)$. Thus the set $[x: T f(x)>\lambda, S f(x)>\mu]$ has this form $(a, \infty)$ or $[a, \infty)$. Let $b>a$. Then $\lambda<T f(b)$, and $\mu<S f(b)$, and so, if (14) holds, we have

$$
\begin{aligned}
\lambda^{r} \mu^{q} & <\left[\int_{0}^{b} k(x, y) f(y) d y\right]^{r}\left[\int_{0}^{b} j(x, y) f(y) d y\right]^{q} \\
& \leq\left[\int_{0}^{b} f(y)^{p} v(y) d y\right]^{(r+q) / p} T_{p^{\prime}} \sigma(b)^{r / p^{\prime}} S_{p^{\prime}} \sigma(b)^{q / p^{\prime}}
\end{aligned}
$$

and (14) gives

$$
\lambda^{r} \mu^{q} \int_{b}^{\infty} w \leq C\left[\int_{0}^{b} f(y)^{p} v(y) d y\right]^{(r+q) / p} \leq C\left[\int_{0}^{\infty} f(y)^{p} v(y) d y\right]^{(r+q) / p} .
$$

Let $b \rightarrow a$ to get (13).

Conversely, fix $x$ and let $E$ be a measurable set $C(0, x)$ for which both

$$
A=T_{p^{\prime}}\left(\sigma \chi_{E}\right)(x) \text { and } B=S_{p^{\prime}}\left(\sigma \chi_{E}\right)(x)
$$

are finite. Set

$$
f(y)=\alpha k(x, y)^{p^{\prime}-1} \sigma(y) \chi_{E}(y) \text { and } g(y)=\beta j(x, y)^{p^{\prime}-1} \sigma(y) \chi_{E}(y) .
$$

Call $h=f+g$. Then if $t \geq x$,

$$
T h(t)>T f(x)=\alpha A, \quad S h(t)>\beta B .
$$

So (13) gives

$$
(\alpha A)^{r}(\beta B)^{q} \int_{x}^{\infty} w \leq C\|h\|_{L^{p}(v)}^{q+r} \leq C\|f\|_{L^{p}(v)}^{q+r}+C\|g\|_{L^{p}(v)}^{q+r} .
$$

But $\|f\|_{L^{p}(v)}=\alpha A^{1 / p}$ and $\|g\|_{L^{p}(v)}=\beta B^{1 / p}$, and so we have

(42) $\quad(\alpha A)^{r}(\beta B)^{q} I^{*} w(x) \leq C\left[\alpha A^{1 / p}+\beta B^{1 / p}\right]^{q+r}$.

Taking $\alpha=1$ and $\beta=\left(A B^{-1}\right)^{1 / p}$ in (42) gives

$$
A^{r / p^{\prime}} B^{q / p^{\prime}} I^{*} w(x) \leq C .
$$

Letting $E$ increase to $(0, x)$ gives (14).

4. Necessary conditions. Let $\alpha, \beta \geq 0$. We investigate the consequences of the second order reduced Opial inequality

$$
\int_{0}^{\infty}\left|T_{\alpha} f(x)\right|^{r}\left|T_{\beta} f(x)\right|^{q} w(x) d x \leq C\left(\int_{0}^{\infty}|f(x)|^{p} v(x) d x\right)^{(q+r) / p} .
$$

The conditions are stated in the following two lemmas.

LEMMA 10. Let $1<q<p<\infty$, and let $r>0$. Let $T$ be a GHO, $\alpha, \beta \geq 0$, and let $(v, w)$ be a weight pair. Then each of the following are consequences of the reduced Opial inequality (43): For each $x>0$,

(44) $\quad T_{\alpha p^{\prime}} \sigma(x)^{\left(r / p^{\prime}\right)(p / q)^{\prime}} \int^{\infty} \sigma(y)\left[T_{\beta q}^{*} w(y) \Gamma \sigma(y)^{q-1}\right]^{(p / q)^{\prime}} d y \leq C$,

(45) $T_{\alpha p^{\prime}} \sigma(x)^{\left(r / p^{\prime}\right)(p / q)^{\prime}} \int_{\infty}^{\infty} w(y)\left[I^{*} w(y) T_{\beta p^{\prime}} \sigma(y)^{p-1}\right]^{(p / q)^{\prime}-1} d y \leq C$,

(46) $I \sigma(x)^{\left(r / p^{\prime}\right)(p / q)^{\prime}} \int_{x}^{\infty} k(y, x)^{\alpha r(p / q)^{\prime}} \sigma(y)\left[T_{\beta q}^{*} w(y) I \sigma(y)^{q-1}\right]^{(p / q)^{\prime}} d y \leq C$,

and

(47) $\quad I \sigma(x)^{\left(r / p^{\prime}\right)(p / q)^{\prime}}$

$$
\int_{x}^{\infty} k(y, x)^{\alpha r(p / q)^{\prime}} w(y)\left[I^{*} w(y) T_{\beta p^{\prime}} \sigma(y)^{p-1}\right]^{(p / q)^{\prime}-1} d y \leq C .
$$

When $q=1$, then (43) implies both (44) and (46). 
LEMMA 11. Let $r>0, q \geq 1,1<p \leq q+r$. Let $T$ be a $G H O, \alpha, \beta \geq 0$, and let $(v, w)$ be a weight pair. Then each of the following are consequences of the reduced Opial inequality (43): For each $x>0$,

$$
\begin{gathered}
T_{\alpha p^{\prime}} \sigma(x)^{r / p^{\prime}} T_{\beta p^{\prime}} \sigma(x)^{q / p^{\prime}} I^{*} w(x) \leq C, \\
I \sigma(x)^{(q+r) / p^{\prime}} T_{\alpha r+\beta q}^{*} w(x) \leq C,
\end{gathered}
$$

and

$$
T_{\beta p^{\prime}} \sigma(x)^{q / p^{\prime}} \operatorname{I\sigma }(x)^{r / p^{\prime}} T_{\alpha q}^{*} w(x) \leq C .
$$

Proof of Lemma 10 . Fix $f, g \geq 0$ with $f$ supported in $(0, x)$, and with $\|f\|_{L^{p}(v)}=\|g\|_{L^{p}(v)}=1$. Applying (43) to $f+g$, we get

$$
C \geq \int T_{\alpha} f(y)^{r} T_{\beta} g(y)^{q} w(y) d y \geq \int_{x}^{\infty} w(y) T_{\beta} g(y)^{q}\left[\int_{0}^{x} k(y, t)^{\alpha} f(t) d t\right]^{r} d y .
$$

Cutting the kernel down to $k(y, x)$ or $k(x, t)$ respectively, we get

$$
C \geq I f(x)^{r} \int_{x}^{\infty} k(y, x)^{\alpha r} w(y) T_{\beta} g(y)^{q} d y
$$

and

$$
C \geq T_{\alpha} f(x)^{r} \int_{x}^{\infty} T_{\beta} g(y)^{q} w(y) d y .
$$

Now take the supremum over such $f$. In (51),

$$
\sup \left\{I f(x)^{r}:\|f\|_{L^{p}(v)}=1, f \geq 0 \text { and supported in }(0, x)\right\}=I \sigma(x)^{r / p^{\prime}} .
$$

Set

Then (51) gives

$$
w_{1}(y)=I_{\sigma}(x)^{r / p^{\prime}} k(y, x)^{\alpha r} w(y) \chi_{(x, \infty)}(y)
$$

$$
\left\|T_{\beta} g\right\|_{L^{q}\left(w_{1}\right)} \leq C\|g\|_{L^{p}(v)}
$$

with the constant $C$ independent of $x$. So, for $q>1,(34)$ and (35) of Stepanov's theorem hold. (34) is

$$
\int_{0}^{\infty} \sigma(t)\left[T_{\beta q}^{*} w_{1}(t) \Gamma \sigma(t)^{q-1}\right]^{(p / q)^{\prime}} d t \leq C
$$

Now, for $t>x$

$$
\begin{aligned}
T_{\beta q}^{*} w_{1}(t) & =I \sigma(x)^{r / p^{\prime}} \int_{t}^{\infty} k(y, t)^{\beta q} k(y, x)^{\alpha r} w(y) d y \\
& \geq k(t, x)^{\alpha r} I \sigma(x)^{r / p^{\prime}} T_{\beta q}^{*} w(x)
\end{aligned}
$$

and so we have (46). Likewise (35) is

$$
\int_{0}^{\infty} w_{1}(t)\left[I^{*} w_{1}(t) T_{\beta p^{\prime}} \sigma(t)^{p-1}\right]^{(p / q)^{\prime}-1} d t \leq C,
$$

and this gives (47).

$$
\text { In (52), } \sup \left(T_{\alpha} f(x)\right)^{r}=T_{\alpha p^{\prime}} \sigma(x)^{r / p^{\prime}} \text {. Taking }
$$

$$
w_{2}(y)=T_{\alpha p^{\prime}} \sigma(x)^{r / p^{\prime}} w(y) \chi_{(x, \infty)}(y)
$$

(52) gives $T_{\beta}: L^{p}(v) \rightarrow L^{q}\left(w_{2}\right)$, and again Stepanov's theorem gives (44) and $(45)$.

Finally, when $q=1$, and when we apply Stepanov's theorem, we get (44) and (46).

Proof of Lemma 11. (48) follows immediately from the weak-type boundedness and Theorem 3. For (49), observe that for $f \geq 0$,

$$
\begin{aligned}
{\left[T_{(\alpha r+\beta q) /(r+q)} f(x)\right]^{r+q} } & =\left[\int_{0}^{x} k(x, y)^{\alpha r /(r+q)} k(x, y)^{\beta q /(r+q)} f(y) d y\right]^{r+q} \\
& \leq\left[\int_{0}^{x} k(x, y)^{\alpha} f(y) d y\right]^{r}\left[\int_{0}^{x} k(x, y)^{\beta} f(y) d y\right]^{q}
\end{aligned}
$$

and so $(43)$ implies

$$
T_{(\alpha r+\beta q) /(r+q)}: L^{p}(v) \rightarrow L^{q+r}(w) .
$$

(49) is simply the Stepanov condition (33). Finally, for $(50)$, we argue as in Lemma 10 , to get $T_{\beta}: L^{p}(v) \rightarrow L^{q}\left(w_{1}\right)$. From the corresponding weak-type inequality, this forces

$$
T_{\beta p^{\prime}} \sigma(y)^{q / p^{\prime}} I^{*} w_{1}(y) \leq C
$$

for all $y>0((53)$ can be proven by following the proof of Theorem 3 with $r=0$. This condition is exactly the first of the Stepanov conditions (32)), and (50) is just (53) when $y=x$. This completes the proof of the second lemma.

5. The special case. We now turn to the inequality (15),

$$
\int_{0}^{\infty}|I f(x)|^{q}|T f(x)|^{r} w(x) d x \leq C\left(\int_{0}^{\infty}|f(x)|^{p} v(x) d x\right)^{(q+r) / p} .
$$

Proof of Theorem 4. (16) and (17) are simply (48) and (49) with $\alpha=1, \beta=0$. For the sufficiency, let $f \geq 0$ be fixed and let $\alpha$ be a large constant. Choose $x_{n}$ so that

$$
\alpha^{n}=\operatorname{If}\left(x_{n}\right)^{g / r} T f\left(x_{n}\right)
$$


Set

$$
I_{n}=\left(x_{n-1}, x_{n}\right) \text { and } J_{n}=\left(0, x_{n-1}\right)
$$

Now

$$
\alpha^{n} \leq C\left(\int_{I_{n}} f\right)^{q / r} \int_{0}^{x_{n}} k\left(x_{n}, y\right) f+C\left(\int_{J_{n}} f\right)^{q / r} \int_{0}^{x_{n}} k\left(x_{n}, y\right) f .
$$

Furthermore, by the triangle inequality for the kernel $k$,

$$
\int_{0}^{x_{n}} k\left(x_{n}, y\right) f \leq \int_{I_{n}} k\left(x_{n}, y\right) f+C k\left(x_{n}, x_{n-1}\right) \int_{J_{n}} f+C T f\left(x_{n-1}\right)
$$

and so (with a different constant $C$ ),

$$
\begin{aligned}
\alpha^{n} \leq & C\left[\left(\int_{I_{n}} f\right)^{q / r} T f\left(x_{n}\right)\right. \\
& \left.+\left(\int_{J_{n}} f\right)^{q / r} \int_{I_{n}} k\left(x_{n}, y\right) f+k\left(x_{n}, x_{n-1}\right)\left(\int_{J_{n}} f\right)^{q / r+1}+\alpha^{n-1}\right] .
\end{aligned}
$$

So long as $\alpha$ is larger than this constant $C$, we can absorb the $\alpha^{n-1}$, to conclude (again with a different constant)

$$
\begin{aligned}
\alpha^{n} \leq & C\left[\left(\int_{I_{n}} f\right)^{q / r} T f\left(x_{n}\right)+k\left(x_{n}, x_{n-1}\right)\left(\int_{J_{n}} f\right)^{q / r+1}\right. \\
& \left.+\left(\int_{J_{n}} f\right)^{q / r} \int_{I_{n}} k\left(x_{n}, y\right) f\right] .
\end{aligned}
$$

Hence, writing $w\left(I_{n}\right)$ for $\int_{I_{n}} w(x) d x$, we obtain

$$
\int(I f)^{q}(T f)^{r} w \leq C \sum_{n} \alpha^{n r} w\left(I_{n+1}\right)
$$

and this splits up into three sums, $I, I I$, and $I I I$, using the decomposition (54). The first of these is

$$
I=\sum_{n} w\left(I_{n+1}\right)\left(\int_{I_{n}} f\right)^{q}\left(\int_{0}^{x_{n}} k\left(x_{n}, y\right) f(y) d y\right)^{r}
$$

Applying Hölder's inequality, we have

$$
\begin{aligned}
I & \leq \sum_{n} w\left(I_{n+1}\right)\left(\int_{I_{n}} f^{p} v\right)^{q / p}\left(\int_{I_{n}} \sigma\right)^{q / p^{\prime}}\left(\int_{0}^{x_{n}} k\left(x_{n}, y\right)^{p^{\prime}} \sigma(y) d y\right)^{r / p^{\prime}}\|f\|_{L^{p}(v)}^{r}\left(x_{n}\right)^{q / p^{\prime}} T_{p^{\prime}} \sigma\left(x_{n}\right)^{r / p^{\prime}}\left(\int_{I_{n}} f^{p} v\right)^{q / p} \\
& \leq\|f\|_{L^{p}(v)}^{r} \sum_{n} I^{*} w\left(x_{n}\right) I \sigma\left(x^{\prime}\right.
\end{aligned}
$$

$$
\begin{aligned}
& \leq C\|f\|_{L^{p}(v)}^{r} \sum_{n}\left(\int_{I_{n}} f^{p} v\right)^{q / p} \text { by }(16), \\
& \leq C\|f\|_{L^{p}(v)}^{r}\left(\sum_{n} \int_{I_{n}} f^{p} v\right)^{q / p}=C\|f\|_{L^{p}(v)}^{r+q},
\end{aligned}
$$

by Minkowski's inequality, valid since $q \geq p$.

The next term, $I I$, is

$$
I I=\sum_{n} w\left(I_{n+1}\right) k\left(x_{n}, x_{n-1}\right)^{r}\left(\int_{J_{n}} f\right)^{q+r} .
$$

Now, if $x \in I_{n+1}$, then

$$
\begin{aligned}
k\left(x_{n}, x_{n-1}\right)^{r}\left(\int_{J_{n}} f\right)^{q+r} & =\left(\int_{J_{n}} k\left(x_{n}, x_{n-1}\right)^{r /(q+r)} f(y) d y\right)^{q+r} \\
& \leq T_{r /(q+r)} f(x)^{q+r} .
\end{aligned}
$$

Thus

$$
I I \leq \int\left(T_{r /(q+r)} f\right)^{q+r} w
$$

and we require

$$
T_{q /(q+r)}: L^{p}(v) \rightarrow L^{q+r}(w)
$$

By Stepanov's theorem, this will follow from (32) and (33), which are

$$
T_{p^{\prime} r /(q+r)} \sigma(x)^{(q+r) / p^{\prime}} I^{*} w(x) \leq C
$$

and

$$
I \sigma(x)^{(q+r) / p^{\prime}} T_{r}^{*} w(x) \leq C .
$$

The latter is (17), and the former follows immediately from (16) and a Hölder's inequality.

This leaves us with $I I I$ to estimate. We get

$$
\begin{aligned}
I I I & =\sum_{n} w\left(I_{n+1}\right)\left(\int_{J_{n}} f\right)^{q}\left(\int_{I_{n}} k\left(x_{n}, y\right) f(y) d y\right)^{r} \\
& \leq \sum_{n} w\left(I_{n+1}\right)\left(\int_{J_{n}} f\right)^{q}\left(\int_{I_{n}} f^{p} v\right)^{r / p}\left(\int_{I_{n}} k\left(x_{n}, y\right)^{p^{\prime}} \sigma\right)^{r / p^{\prime}} .
\end{aligned}
$$

When $p \leq r$, this is handled exactly like $I$. For $p>r$, set $t=(p / r)^{\prime}$ and apply another Hölder's inequality. Then we have

$$
I I I \leq\left(\sum_{n} \int_{I_{n}} f^{p} v\right)^{r / p}\left(\sum_{n} w\left(I_{n+1}\right)^{t}\left(\int_{J_{n}} f\right)^{q t}\left(\int_{I_{n}} k\left(x_{n}, y\right)^{p^{\prime}} \sigma\right)^{r t / p^{\prime}}\right)^{1 / t}
$$


and it will suffice to estimate this last sum $I V$,

$$
I V=\sum_{n} w\left(I_{n+1}\right)^{t}\left(\int_{J_{n}} f\right)^{q t}\left(\int_{I_{n}} k\left(x_{n}, y\right)^{p^{\prime}} \sigma\right)^{r t / p^{\prime}} .
$$

Notice that

$$
\frac{r t}{p^{\prime}}=\frac{p-1}{p / r-1} \geq 1
$$

since $r \geq 1$. We rewrite $I V$ as

$$
\begin{aligned}
I V & =\sum_{n} w\left(I_{n+1}\right)^{t}\left(\int_{J_{n}} f\right)^{q t}\left(\int_{I_{n}} k\left(x_{n}, y\right)^{p^{\prime}} \sigma\right)^{r t / p^{\prime}-1} \int_{I_{n}} k\left(x_{n}, y\right)^{p^{\prime} \sigma} \\
& \leq \sum_{n} w\left(I_{n+1}\right)^{t}\left(\int_{I_{n}} k\left(x_{n}, y\right)^{p^{\prime}} \sigma\right)^{r t / p^{\prime}-1} \int_{I_{n}} k\left(x_{n}, y\right)^{p^{\prime}} \sigma(y) I f(y)^{t q} d y .
\end{aligned}
$$

Set

$$
\begin{aligned}
& u(y)=\sigma(y) I \sigma(y)^{-q t / p^{\prime}-1} . \\
& \int_{I_{n}} k\left(x_{n}, y\right)^{p^{\prime}} \sigma(y) I f(y)^{t q} d y=\int_{I_{n}} k\left(x_{n}, y\right)^{p^{\prime}} u(y) I \sigma(y)^{q t / p^{\prime}+1} I f(y)^{q t} d y \\
& =\int_{I_{n}} I \sigma(y)^{g t / p^{\prime}} I f(y)^{t q} u(y)\left(\int_{0}^{y} k\left(x_{n}, y\right)^{p^{\prime}} \sigma(s) d s\right) \\
& \leq I \sigma\left(x_{n}\right)^{q t / p^{\prime}} T_{p^{\prime}} \sigma\left(x_{n}\right) \int_{I_{n}} I f(y)^{t q} u(y) d y,
\end{aligned}
$$

and so, since $r t / p^{\prime} \geq 1$,

$$
I V \leq \sum_{n}\left[I^{*} w\left(x_{n}\right) I \sigma\left(x_{n}\right)^{q / p^{\prime}} T_{p^{\prime}} \sigma\left(x_{n}\right)^{r / p^{\prime}}\right]^{t} \int_{x_{n}}(I f)^{t q} u \leq C \int(I f)^{t q} u
$$

by (16).

So we can control $I V$ if the Hardy operator $I$ maps $L^{p}(v) \rightarrow L^{t q}(u)$. Since $q \geq p$, we have $t q \geq p$, and so we must verify the Muckenhoupt condition (6),

$$
I \sigma(x)^{t q / p^{\prime}} I^{*} u(x) \leq C
$$

But

$$
I^{*} u(x)=\int_{x}^{\infty} \sigma(y) I \sigma(y)^{-q t / p^{\prime}-1} d y=-\left.\frac{q t}{p^{\prime}} I \sigma(y)^{-q t / p^{\prime}}\right|_{x} ^{\infty} \leq \frac{q t}{p^{\prime}} I \sigma(x)^{-q t / p^{\prime}} .
$$

So (55) holds, and the proof is complete.

Proof of Theorem 5. Again, the implications (i) $\Rightarrow$ (ii) and (i) $\Rightarrow$ (iii) are Lemma 11. For (ii) $\Rightarrow$ (i), we proceed as in the proof of Theorem 4 . The decomposition (54) led to three terms, $I, I I$, and $I I I$. The arguments for $I I$ and $I I I$ are virtually identical, with one exception: To apply (55), we needed $t q \geq p$, and this was trivial in the case of Theorem 4 , whereas now, $q<p$. Still,

$$
t q=\frac{q / p r}{p / r-1}=\frac{q p}{p-r} \geq \frac{q p}{q}=p
$$

since $p \leq q+r$.

What we need is a new argument for term $I$ in the case $q<p$. Set

$$
A=\left\{n: \frac{\alpha^{n}}{2 C}>\left(\int_{I_{n}} f\right)^{q / r} T f\left(x_{n}\right)\right\} .
$$

So, if $n \in A$, then $\alpha^{n}$ is dominated by the last two terms in (54), which led to $I I$ and $I I I$. Thus we can control

$$
\sum_{n \in A} \alpha^{n r} w\left(I_{n+1}\right)
$$

and obviously we can also handle the sum over the terms where $n-1 \in A$. That leaves us to estimate the sum over the set $B$, where

$$
B=\{n: n, n-1 \notin A\} .
$$

Write $I_{B}$ for this sum, $I_{B}=\sum_{n \in B} \alpha^{n r} w\left(I_{n+1}\right)$. When $n \in B$, we have both

$$
\alpha^{n} \leq C\left(\int_{I_{n}} f\right)^{q / r} T f\left(x_{n}\right)
$$

and

$$
\alpha^{n} \leq C\left(\int_{I_{n-1}} f\right)^{q / r} T f\left(x_{n-1}\right) \leq C\left(\int_{I_{n-1}} f\right)^{q / r} T f\left(x_{n}\right) .
$$

Let $0<s \leq q$. We have

$$
\begin{aligned}
& I_{B} \leq C \sum_{n} w\left(I_{n+1}\right)\left(\int_{I_{n}} f\right)^{s}\left(\int_{I_{n-1}} f\right)^{q-s} T f\left(x_{n}\right)^{r} \\
& \leq C \sum_{n} w\left(I_{n+1}\right)\left(\int_{I_{n}} f^{p} v\right)^{s / p}\left(\int_{I_{n-1}} f\right)^{(q-s) / p} \sigma\left(I_{n}\right)^{s / p^{\prime}} \sigma\left(I_{n-1}\right)^{(q-s) / p^{\prime}} T f\left(x_{n}\right)^{r} \\
& \leq C\left[\sum_{n}\left(\int_{I_{n}} f^{p} v\right)^{s / q}\left(\int_{I_{n-1}} f\right)^{(q-s) / q}\right]^{q / p} \\
& \times\left[\sum_{n} w\left(I_{n+1}\right)^{(p / q)^{\prime}} \sigma\left(I_{n}\right)^{\left(s / p^{\prime}\right)(p / q)^{\prime}} \sigma\left(I_{n-1}\right)^{\left((q-s) / p^{\prime}\right)(p / q)^{\prime}} T f\left(x_{n}\right)^{r(p / q)^{\prime}}\right]^{1-q / p} .
\end{aligned}
$$

The first sum handles trivially by Hölder's inequality. For the other sum, we 
choose $s$ so that

$$
\frac{s}{p^{\prime}}\left(\frac{p}{q}\right)^{\prime}=1
$$

Notice that $s=p^{\prime}(1-q / p) \leq p^{\prime}(q-q / p)=q$. Let

$$
\beta=\frac{q-s}{p^{\prime}}\left(\frac{p}{q}\right)^{\prime}
$$

We must estimate

$$
\sum_{n} w\left(I_{n+1}\right)^{(p / q)^{\prime}} \sigma\left(I_{n}\right) \sigma\left(I_{n-1}\right)^{\beta} T f\left(x_{n}\right)^{r(p / q)^{\prime}}
$$

We further decompose this piece into three pieces. The first is

$$
I V=\sum_{n} w\left(I_{n+1}\right)^{(p / q)^{\prime}} \sigma\left(I_{n}\right) \sigma\left(I_{n-1}\right)^{\beta}\left[\int_{I_{n}} k\left(x_{n}, y\right) f(y) d y\right]^{r(p / q)^{\prime}} .
$$

Applying Hölder's inequality,

$$
\begin{aligned}
I V \leq & \sum_{n} w\left(I_{n+1}\right)^{(p / q)^{\prime}} \sigma\left(I_{n}\right) \sigma\left(I_{n-1}\right)^{\beta} \\
& \times\left[\int_{I_{n}} k\left(x_{n}, y\right)^{p^{\prime}} \sigma\right]^{\left(r / p^{\prime}\right)(p / q)^{\prime}}\left(\int_{I_{n}} f^{p} v\right)^{(r / p)(p / q)^{\prime}} .
\end{aligned}
$$

Now,

$$
\frac{1+\beta}{(p / q)^{\prime}}=\frac{s}{p^{\prime}}+\frac{q-s}{p^{\prime}}=\frac{q}{p^{\prime}}
$$

and so,

$$
\begin{aligned}
I V & \leq \sum_{n}\left[I^{*} w\left(x_{n}\right) I \sigma\left(x_{n}\right)^{q / p^{\prime}} T_{p^{\prime}} \sigma\left(x_{n}\right)^{r / p^{\prime}}\right]^{(p / q)^{\prime}}\left(\int_{I_{n}} f^{p} v\right)^{(r / p)(p / q)^{\prime}} \\
& \leq C \sum_{n}\left(\int_{I_{n}} f^{p} v\right)^{(r / p)(p / q)^{\prime}}
\end{aligned}
$$

by (16). But

$$
\frac{r}{p}\left(\frac{p}{q}\right)^{\prime}=\frac{r}{p-q} \geq 1
$$

and so

$$
I V \leq C\left(\int f^{p} v\right)^{r /(p-q)}
$$

We are left with

$$
\sum_{n} w\left(I_{n+1}\right)^{(p / q)^{\prime}} \sigma\left(I_{n-1}\right)^{\beta} \int_{I_{n}} \sigma(x)\left[\int_{J_{n}} k\left(x_{n}, y\right) f(y) d y\right]^{r(p / q)^{\prime}} d x
$$

Now we apply the triangle inequality for $k$. For $x \in I_{n}$,

$$
\int_{J_{n}} k\left(x_{n}, y\right) f(y) d y \leq C\left[k\left(x_{n}, x\right) I f(x)+T f(x)\right] .
$$

This gives us the two other pieces, $V$ and $V I$,

$$
V=\sum_{n} w\left(I_{n+1}\right)^{(p / q)^{\prime}} \sigma\left(I_{n-1}\right)^{\beta} \int_{I_{n}} \sigma(x) k\left(x_{n}, x\right)^{r(p / q)^{\prime}} I f(x)^{r(p / q)^{\prime}} d x
$$

and

$$
V I=\sum_{n} w\left(I_{n+1}\right)^{(p / q)^{\prime}} \sigma\left(I_{n-1}\right)^{\beta} \int_{I_{n}} \sigma(x) T f(x)^{r(p / q)^{\prime}} d x .
$$

For $V$, set

$$
u(y)=\sigma(y) l \sigma(y)^{-\left(r / p^{\prime}\right)(p / q)^{\prime}-1}
$$

Then

$$
\begin{aligned}
V= & \sum_{n} w\left(I_{n+1}\right)^{(p / q)^{\prime}} \sigma\left(I_{n-1}\right)^{\beta} \\
& \times \int_{I_{n}} u(x) I f(x)^{r(p / q)^{\prime}} I \sigma(x)\left[\int_{0}^{x_{n}} k\left(x_{n}, x\right)^{p^{\prime}} \sigma(y) d y\right]^{\left(r / p^{\prime}\right)(p / q)^{\prime}} d x \\
\leq & \sum_{n} I^{*} w\left(x_{n}\right)^{(p / q)^{\prime}} I \sigma\left(x_{n}\right)^{1+\beta} T_{p^{\prime}} \sigma\left(x_{n}\right)^{\left(r / p^{\prime}\right)(p / q)^{\prime}} \int_{I_{n}}(I f)^{r(p / q)^{\prime}} u
\end{aligned}
$$

and so, again using (16), we get

$$
V \leq C \int(I f)^{r(p / q)^{\prime}} u
$$

and this is bounded by (6) and our choice of $u$.

Finally, for $V I$,

$$
V I \leq \int T f(x)^{r(p / q)^{\prime}} \sigma(x) I^{*} w(x)^{(p / q)^{\prime}} I \sigma(x)^{\beta} d x
$$

and so here we need

$$
T: L^{p}(v) \rightarrow L^{r(p / q)^{\prime}}\left[\sigma\left(I^{*} w(I \sigma)^{q-1}\right)^{(p / q)^{\prime}}\right]
$$

which will hold, by Stepanov's theorem, provided both (18) and (19) hold. This completes the proof that (ii) $\Rightarrow$ (i).

The argument for (iii) $\Rightarrow$ (i) is substantially easier. Again, all we have to do is estimate $I$,

$$
I=\sum_{n} w\left(I_{n+1}\right)^{(p / q)^{\prime}} \sigma\left(I_{n}\right)^{\left(q / p^{\prime}\right)(p / q)^{\prime}} T f\left(x_{n}\right)^{r(p / q)^{\prime}}
$$

Now,

$$
w\left(I_{n+1}\right)^{(p / q)^{\prime}}=C \int_{I_{n}} w(y)\left(\int_{y}^{x_{n+1}} w(t) d t\right)^{(p / q)^{\prime}-1} d y \leq C \int_{I_{n}} w I^{*} w^{(p / q)^{\prime}-1}
$$


and so,

$$
I \leq C \int T f(x)^{r(p / q)^{\prime}} w(x)\left[I^{*} w(x) I \sigma(x)^{p-1}\right]^{(p / q)^{\prime}-1} d x
$$

$(20),(21)$, and Stepanov's theorem finish the proof.

\section{The Main Theorem}

Proof of Theorem 6. The necessity is handled by the two lemmas. For the sufficiency, we argue as in the last section. Set

$$
\begin{gathered}
\alpha^{n}=T f\left(x_{n}\right)^{q / r} T_{\gamma} f\left(x_{n}\right), \\
I_{n}=\left(x_{n-1}, x_{n}\right) \text { and } J_{n}=\left(0, x_{n-1}\right) .
\end{gathered}
$$

Then

$$
\begin{aligned}
\alpha^{n} \leq & C\left[\left(\int_{I_{n}} k\left(x_{n}, y\right) f(y) d y\right)^{q / r} T_{\gamma} f\left(x_{n}\right)\right. \\
& +\left(\int_{J_{n}} k\left(x_{n}, y\right) f\right)^{q / r}\left(\int_{I_{n}} k\left(x_{n}, y\right)^{\gamma} f\right) \\
& \left.+\left(\int_{J_{n}} k\left(x_{n}, y\right) f\right)^{q / r} \int_{J_{n}} k\left(x_{n}, y\right) f\right] \\
\leq & C\left[\left(\int_{I_{n}} k\left(x_{n}, y\right) f\right)^{q / r} T_{\gamma} f\left(x_{n}\right)+T f\left(x_{n}\right)^{q / r}\left(\int_{I_{n}} k\left(x_{n}, y\right)^{\gamma} f\right)\right. \\
& +k\left(x_{n}, x_{n-1}\right)^{q / r+\gamma}\left(\int_{J_{n}} f\right)^{q / r+1}+k\left(x_{n}, x_{n-1}\right)^{q / r}\left(\int_{J_{n}} f\right)^{q / r} T_{\gamma} f\left(x_{n-1}\right) \\
& \left.+T f\left(x_{n-1}\right)^{q / r} k\left(x_{n}, x_{n-1}\right)^{\gamma} \int_{J_{n}} f+\alpha^{n-1}\right] .
\end{aligned}
$$

So, with $\alpha$ chosen large enough, we have

$$
\begin{aligned}
C \alpha^{n} \leq & {\left[\left(\int_{I_{n}} k\left(x_{n}, y\right) f\right)^{q / r} T_{\gamma} f\left(x_{n}\right)+T f\left(x_{n}\right)^{q / r}\left(\int_{I_{n}} k\left(x_{n}, y\right)^{\gamma} f\right)\right.} \\
& +k\left(x_{n}, x_{n-1}\right)^{q / r+\gamma}\left(\int_{J_{n}} f\right)^{q / r+1} \\
& +T f\left(x_{n-1}\right)^{q / r} k\left(x_{n}, x_{n-1}\right)^{\gamma} \int_{J_{n}} f \\
& \left.+k\left(x_{n}, x_{n-1}\right)^{q / r}\left(\int_{J_{n}} f\right)^{q / r} T_{\gamma} f\left(x_{n-1}\right)\right] .
\end{aligned}
$$

This decomposition (56) leads to five pieces,

$$
\int(T f)^{q}\left(T_{\gamma} f\right)^{r} w \leq C \sum \alpha^{n r} w\left(I_{n+1}\right) \leq C[I+I I+I I I+I V+V] .
$$

We start with the third piece. For $I I I$,

$$
\begin{aligned}
k\left(x_{n}, x_{n-1}\right)^{q+r \gamma}\left(\int_{J_{n}} f\right)^{q+r} & =\left[\int_{J_{n}} k\left(x_{n}, x_{n-1}\right)^{(q+r \gamma) /(q+r)} f\right]^{q+r} \\
& \leq T_{(q+\gamma r) /(q+r)} f\left(x_{n}\right)^{q+r}
\end{aligned}
$$

and so we need

$$
T_{(q+\gamma r) /(q+r)}: L^{p}(v) \rightarrow L^{q+r}(w)
$$

From Stepanov's theorem, this will hold provided we have (25) as well as

$$
T_{p^{\prime}(q+r \gamma) /(q+r)} \sigma(x)^{(q+r) / p^{\prime}} I^{*} w(x) \leq C .
$$

But Hölder's inequality gives

$$
T_{p^{\prime}(q+r \gamma) /(q+r)} \sigma(x)^{(q+r) / p^{\prime}} \leq T_{p^{\prime}} \sigma(x)^{q / p^{\prime}} T_{\gamma p^{\prime}} \sigma(x)^{r / p^{\prime}}
$$

and so (57) follows from (23).

Next, we estimate $I V$. Here,

$$
\begin{aligned}
& \quad T f\left(x_{n-1}\right)^{q / r} k\left(x_{n}, x_{n-1}\right)^{\gamma} \int_{J_{n}} f \\
& =\int_{J_{n}} k\left(x_{n}, x_{n-1}\right)^{(q+r \gamma) /(q+r)} f\left[\int_{0}^{x_{n-1}} k\left(x_{n-1}, x\right) k\left(x_{n}, x_{n-1}\right)^{(r \gamma-r) /(q+r)} f\right]^{q / r} \\
& \leq T_{(q+r \gamma) /(q+r)} f\left(x_{n}\right)^{1+q / r} \text {, } \\
& \text { and so } I V \text { is controlled exactly like } I I I \text {. } \\
& \quad \text { We now turn to the estimate for } I: \\
& \qquad I=\sum_{n} w\left(I_{n+1}\right)\left(\int_{I_{n}} k\left(x_{n}, y\right) f\right)^{q} T_{\gamma} f\left(x_{n}\right)^{r} \\
& \quad \leq \sum_{n} w\left(I_{n+1}\right)\left(\int_{I_{n}} k\left(x_{n}, y\right)^{p^{\prime}} \sigma(y)\right)^{q / p^{\prime}}\left(\int_{I_{n}} f^{p} v\right)^{q / p} T_{\gamma} f\left(x_{n}\right)^{r} .
\end{aligned}
$$

When $p \leq q, T_{\gamma} f\left(x_{n}\right)^{r} \leq\left(\int f^{p} v\right)^{r / p} T_{\gamma p^{\prime}} \sigma\left(x_{n}\right)^{r / p^{\prime}}$, and $I$ is bounded in the familiar way by (23). So we can focus our attention on the case $q<p$. In that case,

$$
\begin{aligned}
I \leq & \left(\sum_{n} \int_{I_{n}} f^{p} v\right)^{q / p} \\
& \times\left(\sum_{n} w\left(I_{n+1}\right)^{(p / q)^{\prime}}\left(\int_{I_{n}} k\left(x_{n}, y\right)^{p^{\prime}} \sigma\right)^{\left(q / p^{\prime}\right)(p / q)^{\prime}} T_{\gamma} f\left(x_{n}\right)^{r(p / q)^{\prime}}\right)^{1-q / p}
\end{aligned}
$$


and we must estimate this last sum. As in Theorem 5 ,

$$
w\left(I_{n+1}\right)^{(p / q)^{\prime}} \leq C \int_{I_{n}} w\left(I^{*} w\right)^{(p / q)^{\prime}-1}
$$

and so this latter sum is bounded by

$$
C \int\left(T_{\gamma} f\right)^{r(p / q)^{\prime}} w\left[T^{*} w\left(T_{p^{\prime}} \sigma\right)^{p-1}\right]^{(p / q)^{\prime}-1},
$$

which is bounded in turn by Stepanov's theorem, and the conditions (26) and (27).

The estimate for $I I$ is identical, and uses (30) and (31) when $p>r$.

That leaves us only with $V$ to estimate,

$$
V=\sum_{n} w\left(I_{n+1}\right) k\left(x_{n}, x_{n-1}\right)^{q}\left(\int_{J_{n}} f\right)^{q} T_{\gamma} f\left(x_{n-1}\right)^{r}
$$

Set, for $x \in I_{n+1}$,

$$
u(x)=k\left(x_{n}, x_{n-1}\right)^{q} w(x) \text { and } h(x)=x_{n-1} .
$$

Then, in this notation,

$$
V=\int\left[\int_{0}^{h(x)} f\right]^{q}\left[\int_{0}^{h(x)} k(h(x), y)^{\gamma} f(y) d y\right]^{r} u(x) d x
$$

Thanks to the Monotone Convergence Theorem, it will suffice to replace $h$ by an approximating function $g \leq h$ which is strictly increasing, with a differentiable inverse, and with $g\left(x_{n+1}\right)=x_{n-1}$. Set

$$
u_{g}(x)=u\left[g^{-1}(x)\right] \frac{d}{d x} g^{-1}(x) \text {. }
$$

It will suffice to show

$$
\int(I f)^{q}\left(T_{\gamma} f\right)^{r} u_{g} d x \leq C\left(\int f^{p} v\right)^{(q+r) / p}
$$

with this constant $C$ independent of $g$ (and $f$ ). (58) is the special type of Opial inequality studied in the last section, and we need to verify that the weight pair $\left(v, u_{g}\right)$ satisfies the appropriate conditions from Theorems 4 and 5 . To see this, we need a simple estimate: Let $\beta \geq 0$. Then

$$
T_{\beta}^{*} u_{g}(x) \leq T_{\beta+q}^{*} w(x)
$$

To prove (59), fix $x$, and choose $n$ so that $g^{-1}(x) \in I_{n+1}$. Then

$$
\begin{aligned}
T_{\beta}^{*} u_{g}(x) & =\int_{x}^{\infty} k(y, x)^{\beta} u\left[g^{-1}(x)\right]\left(g^{-1}\right)^{\prime}(x) d x=\int_{g^{-1}(x)}^{\infty} k[g(t), x]^{\beta} u(t) d t \\
& \leq \sum_{k \geq n} \int_{I_{k+1}} k[g(t), x]^{\beta} k\left(x_{k}, x_{k-1}\right)^{q} w(t) d t .
\end{aligned}
$$

Now, for $t \in I_{k+1}, g(t) \leq h(t)=x_{k-1}$. So

$$
\begin{aligned}
T_{\beta}^{*} u_{g}(x) & \leq \sum_{k \geq n} \int_{I_{k+1}} k\left(x_{k-1}, x\right)^{\beta} k\left(x_{k}, x_{k-1}\right)^{q} w(t) d t \\
& \leq \int_{x_{n-1}}^{\infty} k(t, x)^{\beta} k\left(t, x_{n-1}\right)^{q} w(t) d t .
\end{aligned}
$$

But $x_{n-1}=g\left(x_{n+1}\right) \geq g\left[g^{-1}(x)\right]=x$, and (59) follows.

Now we can verify the conditions for (58). The first is the weak-type condition (16) from Theorem 4,

(60) $\quad I \sigma(x)^{q / p^{\prime}} T_{\gamma p^{\prime}} \sigma(x)^{r / p^{\prime}} I^{*} u_{g}(x) \leq C$.

But $I^{*} u_{g}(x)=T_{0}^{*} u_{g}(x) \leq T_{q}^{*}(x)$, by (59), and so (60) is simply the condition (24).

The other condition from Theorem 4 is (17),

$$
I \sigma(x)^{(q+r) / p^{\prime}} T_{\gamma r}^{*} u_{g}(x) \leq C,
$$

which follows immediately from (25) and (59).

When $p>q$, we need the two additional conditions (18) and (19) of Theorem 5(ii). These are

(61) $\quad T_{\gamma p^{\prime}} \sigma(x)^{\left(r / p^{\prime}\right)(p / q)^{\prime}} \int_{x}^{\infty} \sigma(y)\left[I^{*} u_{g}(y) I \sigma(y)^{q-1}\right]^{(p / q)^{\prime}} d y \leq C$.

and

(62) $I \sigma(x)^{\left(r / p^{\prime}\right)(p / q)^{\prime}} \int^{\infty} k(y, x)^{\gamma r(p / q)^{\prime}} \sigma(y)\left[I^{*} u_{g}(y) I \sigma(y)^{q-1}\right]^{(p / q)^{\prime}} d y \leq C$.

Replacing $I^{*} u_{g}$ by $T_{q}^{*} w,(61)$ is (28), and (62) is (29). This completes the proof.

\section{References}

[1] M. Axtola, untitled and unpublished manuscript.

[2] P. R. Beesack, Elementary proofs of some Opial-type inequalities, J. Anal. Math. 36 (1979), 1-14.

[3] P. R. Beesack and K. M. Das, Extensions of Opial's inequality, Pacific J. Math. $26(1968), 215-232$.

[4] S. Bloom and R. A. Kerman, Weighted norm inequalities for operators of Hardy type, Proc. Amer. Math. Soc. 113 (1991), 135-141.

[5] D. W. Boyd, Inequalities for positive integral operators, Pacific J. Math. 38 (1971), 9-24.

[6] - Best constants in a class of integral inequalities, ibid. 30 (1969), 367-383.

[7] J. S. Bradley, Hardy inequalities with mixed norms, Canad. Math. Bull. 21 (1978), 405-408. 
[8] W. S. Cheung, Some new Opial-type inequalities, Mathematika 37 (1990), 136-142.

[9] J. D. Li, Opial-type inequalities involving several higher order derivatives, J. Math. Anal. Appl. 167 (1992), 98-110.

[10] F. J. Martín-Reyes and E. T. Sawyer, Weighted inequalities for RiemannLiouville fractional integrals of order one and greater, Proc. Amer. Math. Soc. 106 (1989), 727-733.

[11] V. G. Maz'ja, Sobolev Spaces, Springer, Berlin, 1985.

[12] B. Muckenhoupt, Hardy's inequality with weights, Studia Math. 34 (1972), 31-38.

[13] Z. Opial, Sur une inégalité, Ann. Polon. Math. 8 (1960), 29-32.

[14] B. G. Pachpatte, On Opial type inequalities involving higher order derivatives, J. Math. Anal. Appl. 190 (1995), 763-773.

[15] E. T. Sawyer, Weighted Lebesgue and Lorentz norm inequalities for the Hard operator, Trans. Amer. Math. Soc. 281 (1984), 329-337.

[16] -, A characterization of a two-weight norm inequality for maximal operators, Studia Math. 75 (1982), 1-11.

[17] - A characterization of two weight norm inequalities for fractional and Poisson integrals, Trans. Amer. Math. Soc. 308 (1988), 533-545.

[18] G. J. Sinnamon, Weighted Hardy and Opial-type inequalities, J. Math. Anal. Appl. 160 (1991), 434-445.

[19] V. D. Stepanov, Two-weighted estimates for Riemann-Liouville integrals, Math. USSR-Izy. 36 (1991), 669-681.

[20] - Weighted norm inequalities of Hardy type for a class of integral operators, $\mathrm{Re}$ port/Institute for Applied Mathematics, Khabarovsk, 1992.

[21] G. Talenti, Osservazioni sopra una classe di disuguaglianze, Rend. Sem. Mat. Fis. Milano $39(1969), 171-185$

[22] G. Tomaselli, A class of inequalities, Boll. Un. Mat. Ital. (4) 21 (1969), 622-631.

Department of Mathematics

Siena College

Loudon Road

Loudonville, New York 12211

U.S.A.

\section{Hankel multipliers and transplantation operators}

by

\section{KRZYSZTOF STEMPAK (Wroclaw) and WALTER TREBELS (Darmstadt)}

Dedicated to Professor Satoru Igari on the occasion of his 60th birthday

Abstract. Connections between Hankel transforms of different order for $L^{p}$-functions are examined. Well known are the results of Guy [Guy] and Schindier [Sch]. Further relations result from projection formulae for Bessel functions of different order. Consequences for Hankel multipliers are exhibited and implications for radial Fourier multipliers on Euclidean spaces of different dimensions indicated.

1. Introduction. It is well known that harmonic analysis of radial functions on the Euclidean space $\mathbb{R}^{n}, n \geq 1$, reduces to studying appropriate function spaces on the half-line equipped with the measure $x^{n-1} d x$. The Fourier transform is then replaced by the modified Hankel transform of or$\operatorname{der}(n-2) / 2$. The aim of this paper is to show, among other things, that also studying the non-modified Hankel transform of an arbitrary order $\nu \geq-1 / 2$ within an appropriate weighted setting leads to corresponding results for the Fourier transform of radial functions. This is seen, for instance, in Section 2 where we discuss multiplier results for the modified Hankel transform. It occurs that they are closely related to two transference theorems of Rubio de Francia for the Fourier transform on Euclidean spaces.

Given $\nu \geq-1 / 2$ and $f$, an integrable function on $\mathbb{R}_{+}=(0, \infty)$, its (non-modified) Hankel transform is defined by

$$
\mathcal{H}_{\nu} f(x)=\int_{0}^{\infty}(x y)^{1 / 2} J_{\nu}(x y) f(y) d y, \quad x>0 .
$$

Here $J_{\nu}(x)$ denotes the Bessel function of the first kind of order $\nu[\mathrm{Sz}$ $(1.17 .1)]$. For $\nu=-1 / 2$ or $\nu=1 / 2$ one recovers the cosine and sine trans-

1991 Mathernatics Subject Classification: Primary 42C99; Secondary 44A20.

Key words and phrases: Hankel transform and multipliers, transplantation.

Research of the first author was supported in part by KBN grant \# 2 PO3A 03009. 